\title{
Remdesivir in the COVID-19 Pandemic: An Analysis of Spontaneous Reports in VigiBase During 2020
}

\author{
Elena Rocca ${ }^{1,2} \cdot$ Oskar Gauffin ${ }^{1} \cdot$ Ruth Savage $\mathrm{e}^{1,3,4} \cdot$ Sara Hedfors Vidlin ${ }^{1} \cdot$ Birgitta Grundmark ${ }^{1}$ (I)
}

Accepted: 24 June 2021 / Published online: 10 August 2021

(c) The Author(s) 2021

\begin{abstract}
Introduction The safety profile of remdesivir, conditionally approved for COVID-19, was limited at its 2020 introduction. Adverse drug reactions (ADRs) for medicines are collected in VigiBase, the WHO Global Database of Individual Case Safety Reports (ICSRs).

Objective This study aimed to provide a descriptive analysis of COVID-19 ICSR data focusing on remdesivir, including a disproportionality analysis (DA) of ADRs.

Methods A dedicated algorithm enabled retrieval of all COVID-19 treatment-specific ICSRs. A severity algorithm based on co-reported medicines and symptoms enabled selection of tocilizumab with its well established safety profile as comparator for remdesivir. Descriptive statistics were used for general ICSR demographics for all COVID-19-specific medicines, remdesivir and tocilizumab individually and furthermore to present treatment patterns of medicines co-reported with remdesivir. A COVID-19 indication-focused DA was deployed to minimize confounding from underlying polysymptomatic disease.

Results 14,574 COVID-19-related ICSRs were entered into VigiBase during 2020. Remdesivir was the most common medicine reported. Of 4944 remdesivir ICSRs, where tocilizumab was not co-reported, 93\% described remdesivir as the sole suspect medicine. Sixty percent of ICSRs concerned males, median age was 63 years and the majority originated from the Americas (72\%). In 1089 (21\%) of remdesivir ICSRs, data indicated severe/critical disease. Co-reported medicines peaked during the first 3 days of remdesivir treatment. The DA for the established tocilizumab and the new remdesivir were mainly in line with the safety profiles for both medicines but suggested new safety concerns. The most reported ADRs for remdesivir represented liver dysfunction, kidney injury, death and bradycardia.

Conclusion Global COVID-19-related ADR reporting proved useful in providing information on ADRs as well as on treatment patterns in this patient group. Indication-focused disproportionality analysis, together with the use of a comparator with a known safety profile, proved effective in identifying known safety information and suggested new safety concerns for remdesivir.
\end{abstract}

Elena Rocca

elena.rocca@nmbu.no

$\triangle$ Birgitta Grundmark

birgitta.grundmark@who-umc.org

1 Uppsala Monitoring Centre, Uppsala, Sweden

2 Centre for Applied Philosophy of Science, Norwegian University of Life Sciences, Ås, Norway

3 Division of Health Sciences, New Zealand Pharmacovigilance Centre, University of Otago, Dunedin, New Zealand

4 Department of General Practice, University of Otago, Christchurch, New Zealand 


\section{Key Points}

Remdesivir, a conditionally approved COVID-19 medicine, was, during 2020, the most commonly reported COVID-19 medicine within the global adverse drug reaction reporting into VigiBase, the WHO Global Database of Individual Case Safety Reports (ICSRs).

Remdesivir ICSRs allowed analysis of treatment patterns for co-reported medicines; the patients concerned had a median age of 63 years, were $60 \%$ males, were mostly reported from the Americas and the medicine was, in the majority of the ICSRs, reported as the only suspect medicine.

Indication-focused disproportionality analysis, together with the use of the comparator tocilizumab with a known safety profile, adequately identified known safety information for both remdesivir and tocilizumab and suggested potential safety concerns for remdesivir. The most reported adverse events represented liver dysfunction, kidney injury, death and bradycardia.

\section{Introduction}

In 2020 during the coronavirus disease 2019 (COVID-19) pandemic, global monitoring of adverse drug reactions (ADRs) related to medicinal treatments for the novel disease was performed in VigiBase, the WHO Global Database of Individual Case Safety Reports (ICSRs) [1]. COVID-19 treatments thus monitored included numerous repurposed medicines previously authorized for other indications as well as several novel substances. Remdesivir, one of these novel substances, under study for over a decade but with only scarce pre-pandemic experimental use, quickly gained wide usage. Without solid background data on its safety of the kind normally available for newly authorized medicines, and with rapid and widespread deployment to treat COVID-19 patients through trials and compassionate use, global monitoring and analysis of ICSRs related to the medicine was particularly pertinent to assist in building its safety profile. Causality assessment and analysis of ICSR patterns for remdesivir and other COVID-19 medicines proved unusually challenging. This was on one hand due to the substantial number of concomitant medications used in COVID-19, especially during the first months of the pandemic, when severely ill patients tended to be treated with all clinically sensible therapies available, given the lack of previous experience of the disease. On the other hand, causality assessment was also complicated by the fact that the disease pathophysiology and symptomatology were initially virtually unknown with knowledge only gradually growing during the pandemic.

COVID-19, caused by the novel corona virus (CoV) strain SARS-CoV-2, often manifests as mild to severe pneumonia [2]. Risk factors related to more severe disease include older age, male gender, hypertension, chronic respiratory disease, diabetes, obesity and cardiovascular disease [3, 4]. While respiratory distress syndrome is the primary cause of COVID-19 mortality, sepsis, coagulopathy and other major organ system dysfunctions are also common [5]. The pathogenesis of COVID-19-associated organ dysfunction is still poorly understood.

The antiviral remdesivir was the first approved medicine authorized for the specific treatment indication COVID19 [6]. Preclinical models had shown a broad spectrum of activity against human and zoonotic coronaviruses [7]. Efficacy trials in COVID-19 patients have reported conflicting results [8], where interim results from the multinational WHO Solidarity trial showed that remdesivir conveyed little or no effect on overall mortality, initiation of ventilation and duration of hospital stay [9], while the ACTT-1 trial showed a shorter recovery time with remdesivir compared with placebo [10]. More recent data syntheses confirm that remdesivir does not seem to affect the level of mortality, but appears to reduce the average time to recovery, improves the percentage recovered and reduces serious harm [11]. Based on preliminary evidence, remdesivir was granted emergency approvals for use in hospitalized COVID-19 patients only a few months into the pandemic in regions such as the USA, Japan and the EU $[12,13]$.

At the molecular level, remdesivir is an adenosine analogue prodrug metabolized intracellularly to the pharmacologically active remdesivir triphosphate (RTP). RTP competes with endogenous adenosine triphosphate (ATP), resulting in abnormal replication of viral RNA with loss of further replication. Preliminary pharmacokinetic data indicates that RTP is a substrate of cytochrome P450 enzymes and is extensively metabolized, although the enzymes responsible for the hydrolytic metabolism are still unknown. Remdesivir and RTP are predominantly excreted in urine and the terminal half-life is approximately 24 hours [14].

The knowledge of remdesivir's safety profile is still limited but increasing, for example, through analyses of reported ICSRs and further clinical trials. Some analyses of remdesivir pharmacovigilance data focussing on specific [15-17] or general [18] adverse drug reactions have recently been published based on early data. Acknowledged potential harms include hypersensitivity, infusion-related and anaphylactic reactions (rare), transaminase elevation (very common), nausea (common), headache (common), rash (common) and renal impairment (precaution) [6]. 
Interactions, including risk of decreased antiviral activity when co-administrated with chloroquine and hydroxychloroquine, are also known [6].

\section{The WHO Programme for International Drug Monitoring-VigiBase}

All 142 National Centres (NCs) of the WHO Programme for International Drug Monitoring (PIDM) share ICSRs collected nationally into VigiBase [19], making the more than 24 million globally collected ICSRs (January 2021) accessible for analysis to all NCs. Uppsala Monitoring Centre (UMC), the WHO Collaborating Centre for International Drug Monitoring, holds and maintains the database and undertakes statistical and clinical analyses for the detection of global safety signals within it. A signal, in this context, is described as a hypothesis of a previously undetected or incompletely documented risk associated with a medicine, with various levels of evidence and arguments to support it [19].

In January 2020, the first ICSR related to COVID-19 treatment was shared into VigiBase. Regular reviews of COVID-19 reporting were subsequently produced for the NCs of the WHO-PIDM throughout 2020 [1].

ICSRs concern suspected ADRs and causality assessment to determine the likelihood of the reported event being caused by the medicine in question is performed by recipients of the ICSRs, such as National Centres of Pharmacovigilance using one of a few standard methodologies [20]. These utilize background information on the medicine and on the disease under treatment. In addition, case descriptions are investigated for support for the suspicion or alternative explanations. However, standard methodologies for causality assessment have been particularly difficult to employ during the COVID-19 pandemic. This is due to at least three reasons: (i) the novelty and apparent multi-organ effects associated with the disease itself; (ii) a high rate of incompleteness of reporting into VigiBase, in part due to apparent time pressure and emergency situations of the reporting; and (iii) multiple concomitant medicines. The present study is motivated by these difficulties and intended as a support for the analysis of remdesivir ADR reporting.

\section{Aim}

Our aim is to present an overview of the global ADR reporting pattern for remdesivir, based on a descriptive and comparative analysis of data present in ICSRs related to COVID19 and shared into VigiBase during 2020. We further aim at a comprehensive description of the information shared through spontaneous reporting to give an impression of treatment patterns during the first year of the pandemic. Because of this, ICSR data analysed includes general demographic information and patterns of co-reported medicines initiated with a close time relationship with the reporting and hence also with the disease. Finally, we aim at complementing available remdesivir safety data through the analysis of real-world evidence. Specifically, we are going to explore causality of the reported remdesivir-ADR combinations using an indication-focused disproportionality analysis. In parallel, we will run the same analysis with a comparable COVID-19 medicine with a well-established safety profile and use the results to test the validity of the method.

\section{Methods}

The dedicated methodology used for the retrieval and analysis of COVID-19-specific ICSRs has been presented earlier [21]. VigiBase was screened for COVID-19-specific ICSRs, that is, those in which the indication for at least one medicine was noted as COVID-19. Initially, during the pandemic, coding terminologies did not contain COVID-19-specific terms. Therefore, relevant ICSRs were extracted from VigiBase by scanning incoming ICSRs for indications for each reported medicine, the free-text narratives and the laboratory test result fields through the in-house developed algorithm [21]. All medicines in the extracted ICSRs were manually classified, at the time of their first being reported, by two independent assessors as either COVID-19-specific medicines or non-COVID-19-specific medicines. COVID-19-specific medicines were identified from scientific literature and clinical trials databases as medicines that were being used to treat COVID-19 infection itself and/or medicines with an existing theoretical hypothesis of a mechanism of action for the disease. Non-COVID-19-specific medicines were those that did not satisfy these criteria. For instance, azithromycin was hereby classified as a COVID-19-specific medicine while other antibiotics were not [22], but the latter were used for patients with COVID-19 to manage the complications. COVID-19 ICSRs were included in this study if they entered VigiBase between 01 January 2020 and 31 December 2020.

General demographics, reporting dates, co-reported medicines and reported ADRs as coded with the Medical Dictionary for Regulatory Activities (MedDRA ${ }^{\circledR}$, the international medical terminology developed under the auspices of the International Council for Harmonisation of Technical Requirements for Pharmaceuticals for Human Use) preferred terms (PTs) were used for a descriptive comparison of remdesivir ICSRs with other COVID-19-specific medicine ICSRs, including a timeline for co-reported medicines. As a measure of the level of clinically relevant structured information within the ICSRs (including presence of free-text narrative), the vigiGrade completeness score [23] was used, 
the bounds of which are 0-100, where higher scores denote a higher level of completeness of the data in the ICSR.

For a comparative analysis of remdesivir's reporting patterns, a clinically relevant comparator among the COVID19-specific medicines with an already well-established safety profile was chosen. This was based on a comparison of the level of severity of the COVID-19 infection derived from information in the ICSRs. The proportion of ICSRs describing a severe or critical level of COVID-19 [24] was identified for each of the COVID-19-specific medicines, where all glucocorticoids and heparin-class substances were counted collectively in their respective groups. This was done by scanning the structured fields of ICSRs for the presence of one or more of the following indicators implying severe disease: PTs hypoxia/oxygen saturation decreased; medicines in any of the ATC groups M03A Muscle Relaxants; N01A General Anaesthetics; C01CA Adrenergic and Dopaminergic Agents; and albumin/sodium chloride infusion (see Supplementary Table 1 in the Electronic Supplementary Material [ESM]). Tocilizumab, glucocorticoids and ascorbic acid had the most similar profile to remdesivir for clinical severity of the COVID-19 infection. Tocilizumab was chosen as the preferred comparator as, like remdesivir, it has a specific proposed primary mode of action against COVID-19. Demographic data was compared between the following groups: all COVID-19-related ICSRs, ICSRs with remdesivir, ICSRs with tocilizumab and ICSRs with remdesivir in combination with tocilizumab.

Longitudinal trends are presented for the arrival date at national centres of remdesivir, tocilizumab and all COVID19-related ICSRs.

To describe co-treatment patterns, medicines co-reported in remdesivir ICSRs were analysed. The co-reported medicines were classified as COVID-19 specific/non-COVID-19 specific, as described above. Among remdesivir ICSRs, those containing complete dates of initiation and discontinuation of both remdesivir and the co-reported medicines were included in a longitudinal visualization of the day-by-day co-reporting pattern of medicines in relation to remdesivir treatment.

To provide a quantitative perspective on reported ADRs, an indication-focused disproportionality analysis (DA) was performed [25] for remdesivir and the clinically chosen comparator tocilizumab. Confounding by the underlying disease was addressed by a reduced-background approach [26], using the ICSRs identified with a COVID-19 indication as background. An ADR was considered statistically disproportionate at a credible level of $95 \%$ when the quantile $\mathrm{IC}_{025 \text { CoviD }}$ exceeded zero. The DA was used for remdesivir and tocilizumab to complement the analysis of the ADR reporting in relation to their respective EU safety labelling $[6,27]$.

All analyses were made using the R environment [28].

\section{Results}

\subsection{General Description of Remdesivir ADR Reporting in VigiBase}

Remdesivir was the most commonly reported COVID19-specific medicine, appearing in 5299 of the total 14,574 extracted COVID-19-related ICSRs (35.4\%). The complete list of the reported COVID-19-specific medicines and their respective number of ICSRs is available as Supplementary Table 2 (see ESM). Concomitant medicines or MedDRA PTs indicating severe or critical COVID-19 infection were present in 1089 (21\%) of remdesivir ICSRs and $214(16 \%)$ of the tocilizumab ICSRs (Supplementary Table 1, see ESM).

As noted in Table 1, monotherapy was reported to a similar extent for remdesivir (40\%), tocilizumab (41\%) and for all medicines (38\%). Reported remdesivir monotherapy ICSRs (i.e. reports lacking structured data on any co-reported medicines) were in general also found to be incomplete with regard to other regularly reported information such as demographic data and dates.

The median completeness score was lower for both remdesivir (57) and tocilizumab ICSRs (36) compared with the median completeness score of all COVID-19 ICSRs (63). The median completeness score for all ICSRs in VigiBase is 44 (not shown in table).

The median number of reported medicines and reported reactions in the ICSRs were similar in the subgroups. Notably, remdesivir was reported as the single (only) suspected medicine in $93 \%$ of the ICSRs, more often than tocilizumab (57\%) and all the COVID-19-specific medicines (70\%). Males were represented more often than females for both the individual medicines and all COVID-19 medicines. The median patient age was higher in remdesivir ICSRs compared with the comparator ICSRs.

The global reporting for remdesivir and all COVID-19 medicines during the study period came predominantly from the WHO region of the Americas with $72 \%$ and $51 \%$ respectively, the European region $(15 \%, 29 \%)$ and the SouthEast Asia region $(7 \%, 11 \%)$, while none of the remdesivir ICSRs and $1 \%$ of all COVID-19-related ICSRs came from the WHO African region. For comparison, the proportion of VigiBase ICSRs for any medicine during the same period was 35\% from the WHO region of the Americas, 22\% from the European region, 3.5\% from the South-East Asia region and $1 \%$ from the WHO African region (not shown in table).

The longitudinal trends of ICSR reporting of remdesivir, tocilizumab and all COVID-19 medicines during the study period are visualized in Fig. 1. The very first COVID-19-related ICSR was reported to an NC on 30 January 2020 and was indeed a remdesivir report. Global reporting between January and April was dominated by medicines from the 
Table 1 Overall demographics of COVID-19-specific ICSRs

\begin{tabular}{|c|c|c|c|c|c|c|c|c|}
\hline & All & & Remdesivir & & Tocilizumab & & $\begin{array}{l}\text { Remdesivir } \\
\text { tocilizumab }\end{array}$ & \\
\hline & $N$ & $\%$ & $N$ & $\%$ & $N$ & $\%$ & $N$ & $\%$ \\
\hline \multicolumn{9}{|l|}{ Report characteristics } \\
\hline Total $N$ & 14,574 & 100 & 4944 & 100 & 993 & 100 & 355 & 100 \\
\hline Monotherapy & 5468 & 38 & 1989 & 40 & 411 & 41 & - & - \\
\hline Median completeness score (Q1-Q3) & $63(40-92)$ & & $57(44-63)$ & & $36(26-70)$ & & $57(38-65)$ & \\
\hline Median $N$ drugs (Q1-Q3) & $3(1-5)$ & & $3(1-8)$ & & $3(1-7)$ & & $8(4-15)$ & \\
\hline Median N PTs (Q1-Q3) & $1(2-2)$ & & $1(1-3)$ & & $3(2-3)$ & & $2(1-4)$ & \\
\hline Suspected $N$ & 14,547 & 100 & 4858 & 98 & 847 & 85 & - & - \\
\hline Single suspected & 10,162 & 70 & 4619 & 93 & 562 & 57 & - & - \\
\hline Serious & 7717 & 53 & 3461 & 70 & 715 & 72 & 306 & 86 \\
\hline Fatal & 2093 & 14 & 1070 & 22 & 285 & 29 & 103 & 29 \\
\hline \multicolumn{9}{|l|}{ Sex } \\
\hline Female & 5691 & 39 & 1814 & 37 & 231 & 23 & 104 & 29 \\
\hline Male & 7928 & 54 & 2982 & 60 & 553 & 56 & 228 & 64 \\
\hline Unknown & 955 & 7 & 148 & 3 & 209 & 21 & 23 & 6 \\
\hline \multicolumn{9}{|l|}{ Age } \\
\hline Median (Q1-Q3) & $57(42-70)$ & & $63(49-74)$ & & $60(48-67)$ & & $62(47-71)$ & \\
\hline $0-17$ years & 280 & 2 & 70 & 1 & 15 & 2 & 6 & 2 \\
\hline $18-44$ years & 3363 & 23 & 722 & 15 & 124 & 12 & 55 & 15 \\
\hline $45-64$ years & 4518 & 31 & 1455 & 29 & 342 & 34 & 107 & 30 \\
\hline $65-74$ years & 2420 & 17 & 909 & 18 & 189 & 19 & 94 & 26 \\
\hline$>74$ years & 1984 & 14 & 962 & 19 & 66 & 7 & 40 & 11 \\
\hline Unknown & 2009 & 14 & 826 & 17 & 257 & 26 & 53 & 15 \\
\hline \multicolumn{9}{|l|}{ WHO region } \\
\hline African Region & 79 & 1 & 1 & 0 & 23 & 2 & 2 & 1 \\
\hline Eastern Mediterranean Region & 1154 & 8 & 49 & 1 & 32 & 3 & 2 & 1 \\
\hline European Region & 4298 & 29 & 739 & 15 & 438 & 44 & 36 & 10 \\
\hline Region of the Americas & 7395 & 51 & 3563 & 72 & 359 & 36 & 294 & 83 \\
\hline South-East Asia Region & 993 & 7 & 529 & 11 & 90 & 9 & 15 & 4 \\
\hline Western Pacific Region & 655 & 4 & 63 & 1 & 51 & 5 & 6 & 2 \\
\hline
\end{tabular}

'All' refers to the total number of VigiBase ICSRs with COVID-19-specific treatments. The columns headed 'Remdesivir' and 'Tocilizumab' show ICSRs where the corresponding medicine is reported without the other. The column 'Remdesivir and tocilizumab' shows ICSRs where both medicines are reported. All total counts ('Total N') refer to the number of ICSRs where the medicine was reported as suspected, interacting, or concomitant in relation to the reported event(s), while 'Suspected N' refers to the number where it was reported as suspected or interacting. Fatal refers to the number of ICSRs with a fatal outcome (please note that the causal relationship is not evaluated). Q1 and Q3 refers to the first and third quartile. 'Serious' refers here to the reported event according to the CIOMS definition [29]

ICSRs Individual Case Safety Reports

WHO-initiated Solidarity trial; besides remdesivir, hydroxychloroquine, chloroquine, azithromycin and lopinavir/ritonavir were also reported. After April, reporting of remdesivir rapidly escalated, constituting a majority of the reported COVID-19-specific medicines by the end of 2020. The apparent decline in reporting in December in the figure is at least partly an artefact caused by significant and varying delay globally between an ADR occurring in a patient and the ICSR being submitted to VigiBase.

\subsection{Treatment Patterns; Medicines Co-Reported with Remdesivir}

Sixty percent of remdesivir ICSRs contained at least one co-reported medicine. In the ICSRs, several COVID19-specific medicines were used concomitantly with remdesivir (Table 2). One third contained co-reporting of antithrombotic agents and/or glucocorticoids. Fourteen percent of patients were co-medicated with azithromycin. Regarding non-COVID-19-specific medicines co-reported with remdesivir (Table 3), 30\% of patients were treated 


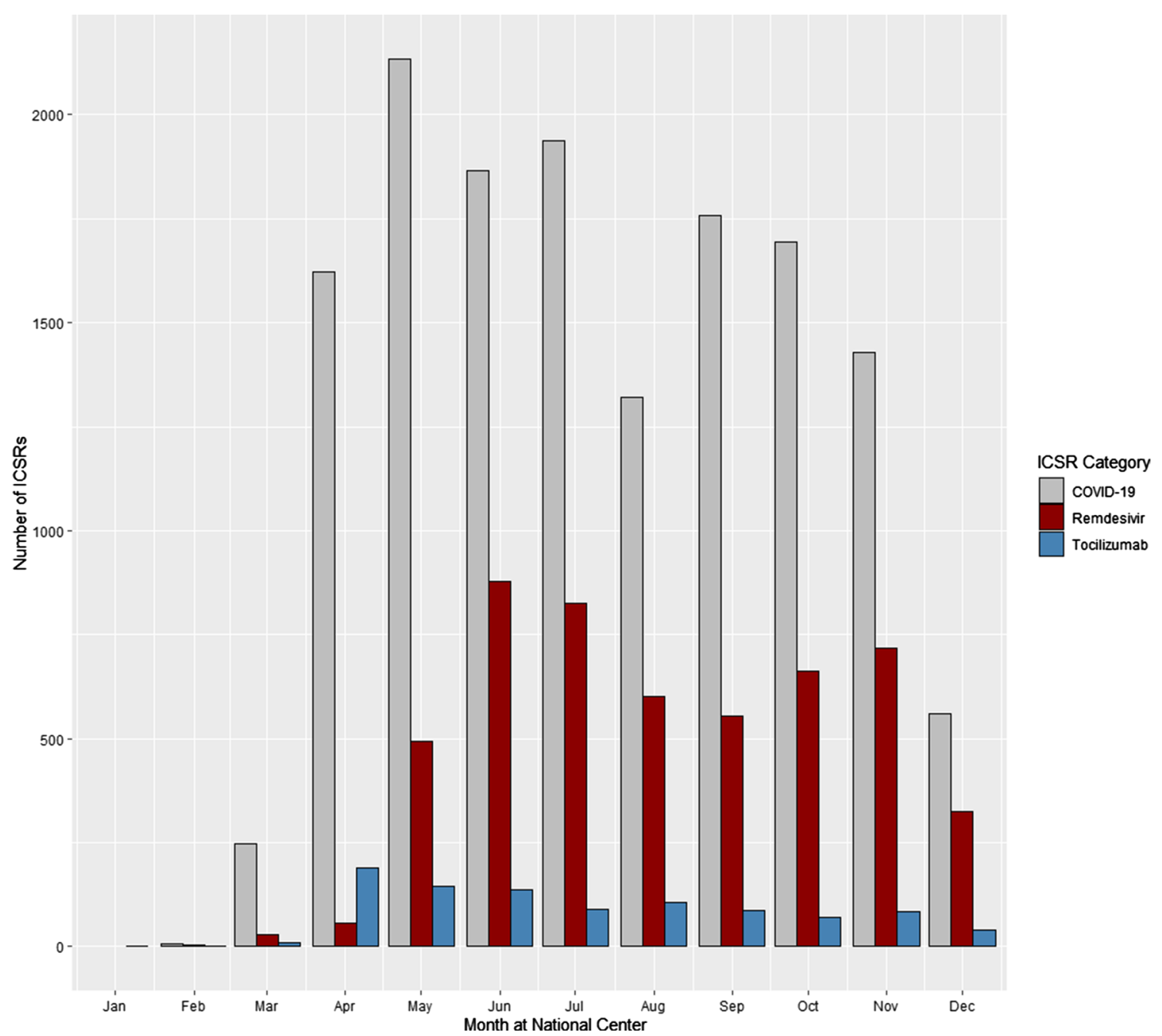

Fig. 1 Longitudinal trends of Individual Case Safety Report (ICSR) reporting of remdesivir, tocilizumab and all COVID-19-specific medicines (including remdesivir and tocilizumab) to the respective

with at least one concomitant systemic antibacterial and $25 \%$ with a concomitant analgesic. Antidiabetics were reported in $10 \%$ of remdesivir ICSRs. The proportion of ICSRs with complete treatment dates were in the same order of magnitude for all medicines except oxygen, where complete treatment dates were rare. For expanded lists of co-medications reported with remdesivir, please see Supplementary Tables 3 and 4 (see ESM). For a general overview of co-medication frequencies among the mostreported COVID-19-specific medicines, see Supplementary Table 5 in the ESM.

Figure 2 shows the longitudinal visualization of the day-by-day co-reporting pattern of medicines in relation to remdesivir treatment (based on ICSRs with treatment date information). Among these, both COVID-19-specific and non-specific co-medications were started up to 2 weeks prior to remdesivir initiation, increasing in frequency until national pharmacovigilance centres during 2020. Counts include medicines reported as suspected or interacting. Note that remdesivir and tocilizumab bars are not mutually exclusive (Table 1)

reaching a maximum on the day of remdesivir initiation and days $1-2$ thereafter.

\subsection{Reported Reactions}

As presented in Fig. 3, for tocilizumab the reported PTs hypofibrinogenaemia, intestinal perforations, infections and hypertriglyceridaemia are examples of specific PTs among many present in the medicine's label. These are also reported to a high and disproportionate extent for tocilizumab compared with other COVID-19-specific medicines. Increased hepatic enzymes/transaminases, dyspnoea and rashes are among labelled PTs not reported disproportionately compared with the same background.

As shown in Fig. 3, for remdesivir, as for tocilizumab, there is for the most part concordance between the disproportionately reported PTs and the labelling, such as 
Table 2 COVID-19-specific co-medications for remdesivir

\begin{tabular}{lllll}
\hline ATC & ATC name (name, N of ICSRs for top drug in ATC group) & N ICSRs & Remdesivir \% & $\begin{array}{l}\text { Com- } \\
\text { plete } \\
\text { dates } \%\end{array}$ \\
\hline B01 & Antithrombotic agents (Heparin group, $N=1637)$ & 1748 & 33 & 35 \\
H02 & Corticosteroids for systemic use (Glucocorticoids, $N=1689)$ & 1689 & 32 & 39 \\
J01 & Antibacterials for systemic use (Azithromycin, $N=766)$ & 766 & 14 & 46 \\
A11 & Vitamins (Ascorbic acid, $N=412)$ & 527 & 10 & 34 \\
L04 & Immunosuppressants (Tocilizumab, $N=355)$ & 394 & 7 & 46 \\
A12 & Mineral supplements (Zinc, $N=327)$ & 332 & 6 & 41 \\
P01 & Antiprotozoals (Hydroxychloroquine, $N=224)$ & 228 & 4 & 28 \\
R03 & Drugs for obstructive airway diseases (Montelukast, $N=54)$ & 94 & 2 & 27 \\
J05 & Antivirals for systemic use (Lopinavir; Ritonavir, $N=42)$ & 91 & 2 & 35 \\
L01 & Antineoplastic agents (Rituximab, $N=10)$ & 24 & 0 & 33 \\
\hline
\end{tabular}

Top ten COVID-19-specific medicines co-reported with remdesivir grouped by Anatomical Therapeutic Chemical (ATC) classification, level 2. Name of each ATC group is followed by the most frequently reported medicine in that group, e.g. 'A11 Vitamins' contains 412 ICSRs with ascorbic acid out of the total 527 ICSRs with 'A11 Vitamins'. Column 'N ICSRs' displays the total count of remdesivir ICSRs with a medicine in each ATC group. Column 'Remdesivir \%' displays the percentage of ICSRs containing medicines from each ATC group, out of all remdesivir ICSRs $(N=5299)$

Column 'Complete dates \%' specifies the percentage of ICSRs with complete dates for initiation and discontinuation for remdesivir and at least one co-reported medicine from the indicated ATC group used in Fig. 2. Note that ATC groups have been merged, e.g. all corticosteroids reported are here merged into 'ATC H02'. For completeness, see Supplementary Tables 3 and 4 in the Electronic Supplementary Material listing the co-reporting frequencies of specific medicines belonging to each ATC group. ICSRs Individual Case Safety Reports

\begin{tabular}{lllll}
\hline ATC & ATC name (Top drug in class, N for top drug) & N ICSRs & Remdesivir $\%$ & $\begin{array}{l}\text { Com- } \\
\text { plete } \\
\text { dates } \%\end{array}$ \\
& & & 48 \\
J01 & Antibacterials for systemic use (Ceftriaxone, $N=748)$ & 1592 & 30 & 37 \\
N02 & Analgesics (Paracetamol, $N=702)$ & 1306 & 25 & 33 \\
A02 & Drugs for acid-related disorders (Pantoprazole, $N=467)$ & 1041 & 20 & 41 \\
N05 & Psycholeptics (Midazolam, $N=335)$ & 778 & 15 & 43 \\
N01 & Anesthetics (Propofol, $N=497)$ & 700 & 13 & 42 \\
C01 & Cardiac therapy (Norepinephrine, $N=481)$ & 679 & 13 & 4 \\
V03 & All other therapeutic products (Oxygen, $N=567)$ & 621 & 12 & 37 \\
A10 & Drugs used in diabetes (Insulin lispro, $N=258)$ & 530 & 10 & 43 \\
C03 & Diuretics (Furosemide, $N=432$ ) & 519 & 10 & 36 \\
R03 & Drugs for obstructive airway diseases (Salbutamol, $N=328)$ & 502 & 9 & \\
\hline
\end{tabular}

Analogous to Table 2, for non-COVID-19-specific medicines

ATC Anatomical Therapeutic Chemical, ICSRs Individual Case Safety Reports
Table 3 Non-COVID-19specific co-medications for remdesivir

\section{Discussion}

Our study of data from the global ADR reporting during 2020 of the COVID-19 pandemic, with specific focus on remdesivir, offers a unique view of the medicine's global clinical utilization and of the safety concerns communicated by the users worldwide via ICSRs. 


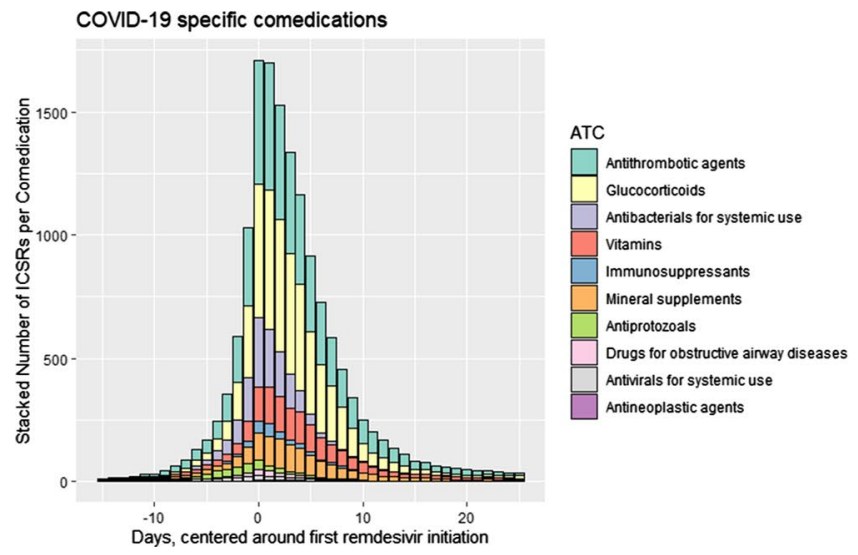

Fig. 2 Plot of the frequency of the top ten co-reported COVID19-specific medicines across time grouped by Anatomical Therapeutic Chemical (ATC) classification, level 2. Day zero is the day of first

The main findings of the study are as follows: (i) remdesivir ICSRs reported mainly from the Americas and Europe constituted the largest proportion of COVID-19-related ADR reporting during 2020; (ii) the median age of the patients in remdesivir ICSRs was higher than for other COVID-19 medicines and the majority of ICSRs for all COVID-19-specific medicines concerned men; (iii) the longitudinal pattern of reporting of medicines with a COVID-19 indication in VigiBase included the early and rapid appearance and increase of reporting for remdesivir during the pandemic; and finally, (iv) performing an indication-based disproportionality analysis, that is, using a background based on the indication for treatment (i.e. COVID-19) to minimize the confounding by indication, proved useful. The medicine-ADR combinations highlighted by the method coincided to a relevant degree with the acknowledged safety profiles of both remdesivir and the comparator medicine tocilizumab, and some new safety concerns related to remdesivir could be suggested.

To our knowledge, studies based on global COVID-19 ICSRs data related to remdesivir [15-18] mostly have a narrow focus, analysing specific medicine-ADR combinations using a smaller dataset compared with the present study. Our study provides a broader descriptive picture of the utilization of remdesivir beyond the reported ADRs. Furthermore, the data capture and analyses in our study, including the indication-focused DA, are more elaborate and provide more precise results, thereby adding important knowledge to what has been published so far in relation to remdesivir.

The comparatively high level of remdesivir reporting within the COVID-19-related reporting could be an indicator of the medicine's widespread use in Europe and the Americas, from where most of the reporting originated, and is also probably due to the novelty of remdesivir triggering closer monitoring and ADR reporting compared with the chosen comparator tocilizumab and other COVID-19 medicines,

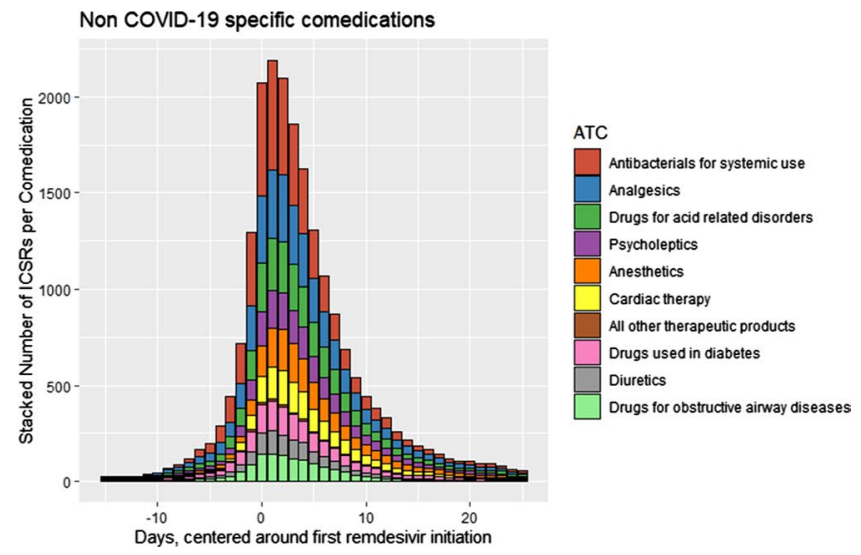

initiation of remdesivir. Note that ATC groups with the same names in the two subgraphs refer to COVID-19-specific and non-specific medicines, see Tables 2 and 3. ICSRs Individual Case Safety Reports

all with well-established pre-pandemic safety profiles. The global geographic reporting pattern for remdesivir, dominated by the Americas and Europe and with little contribution from the African continent, is more pronounced for the COVID-19 reporting than for VigiBase as a whole and may support the observation that global differences in medicines availability and quality of healthcare have become more pronounced during the pandemic [30]. The finding may also partly be influenced by variable incidence of the disease in different countries and regions over time. The comparatively higher median age of the remdesivir ICSR patients is not surprising given that the medicine is indicated for hospitalized patients, and that hospitalization in COVID-19 has been more frequent in elderly patients [31]. Our finding that ICSRs related to remdesivir and all COVID-19-specific medicines more often concerned males than females is also consistent with earlier findings [21].

The results of the indication-based disproportionality analysis for tocilizumab are largely consistent with the known safety profile of the medicine. Two exceptions are the increased hepatic enzymes/transaminases and infusionrelated reactions, which are not reported disproportionately although presented in the label as common. This may either reflect less reporting for known non-serious reactions with an established medicine in critical disease, or be partly a masking phenomenon by the higher reporting of these reactions in relation to remdesivir.

Similarly, reactions labelled for remdesivir are to a large extent also found to be disproportionately reported in our analysis. These findings, in keeping with what is known, suggest that attention should be paid to the unexpected disproportionate reporting for remdesivir including cardiac conditions, product administration error and respiratory conditions, as well as ICSRs of severe hepatic reactions in the context of remdesivir's known effect of increasing hepatic 
transaminases, and finally, ICSRs of severe renal injury, information on which has been communicated to the NCs of the WHO-PIDM via UMC's regular COVID-19 reviews throughout 2020 [1]. Of note, the severity of hepatic events reported differs from the labelling and from the reporting for the other COVID-19-specific medicines. While there are several potential explanations for this besides the existence of a true causal association between remdesivir and severe hepatic reactions, including acute hepatic failure, a more thorough investigation of the matter is needed. This may include analysis of the clinical relevance of the time to onset reported in the ICSRs, of presence of relevant patterns of increase and decrease of biomarkers for liver injury and of findings of response to acetylcysteine. Supportive findings such as these may support suggestions of cases of druginduced liver injury (DILI) where remdesivir is one of the contributing factors [32].

Some of the reported PTs may still, despite the use of the indication-focused disproportionality analysis, suffer from confounding from the underlying disease. Although confounding by the disease and differences in disease severity were addressed by a focused background approach and by using tocilizumab as comparator, it remains difficult to discriminate whether reported PTs (e.g. respiratory terms) refer to the underlying COVID-19 infection or to a medicine reaction such as respiratory manifestations of an allergic or hypersensitivity reaction. Thus, a careful clinical analysis is needed to investigate ICSRs of possible remdesivir-specific reactions.

Another notable observation is the unexpectedly high proportion of ICSRs in which remdesivir is reported as monotherapy. We are hesitant to assume that the monotherapy reported described clinical reality, as the clinical context made it unlikely. In general, we believe that lack of coreported medicines was more likely due to incompleteness of reporting, possibly due to staff having limited time to report concomitant medicines, rather than to actual monotherapy.

The ICSR data on co-reported medications initiated with a close time relationship to remdesivir provides real-world evidence that during the first year of the pandemic the clinical approach to severely ill COVID-19 patients included a massive co-treatment arsenal of medicines, both COVID-19 specific and non-COVID-19 specific. The longitudinal data in the ICSRs with multiple medications initiated around the start of remdesivir supports the described pattern of severe COVID-19 development where after a week from symptom start some patients deteriorate, becoming hypoxic and necessitating more intensive treatment, including remdesivir [33]. Both clinical experience $[2,3]$ and positive study results with the use of antithrombotic agents and/or glucocorticoids in COVID-19 patients $[34,35]$ are reflected within the remdesivir ICSRs, these medicines being the most commonly co-reported medicines during the study period. Among the
non-COVID-19-specific co-reported medications, systemic antibacterials and analgesics were unsurprisingly the most common. The level of antidiabetic medicines co-reported in the remdesivir ICSRs could either be a marker for preexisting diabetes as a risk factor, an indication for a metabolic disturbance due to the disease, or an indicator of active blood glucose control in an intensive care unit [36]. Neither diabetes nor hyperglycaemia was reported as an ADR for remdesivir to any significant extent.

A strength of the study is the global and unrestricted nature of the data. Further, the methodology used to focus the inclusion of ICSRs for analysis only to those with a COVID-19 indication makes it unique where the indication-based disproportionality analysis performed allows the influence of COVID-19 disease to be minimized; this is particularly relevant for polysymptomatic COVID-19 disease. As far as we know, this is the first study to use this methodology, with its advantages in relation to hypotheses generated, compared with studies not using it. Another strength of this paper is the selection of a clinically relevant suitable comparator, based on reported clinical use and with a known safety profile. This has served as a validation of the DA findings.

Limitations of this study include the inherent incompleteness of spontaneously reported ICSR data, for example, regarding dates and co-reported medicines as well as the obvious underreporting of ADRs in general, so that reallife generalizations on frequencies have not been assumed. This particular pandemic situation with a polysymptomatic disease and often numerous co-medications complicates any causality assessment of reported ADRs in relation to a novel medicine such as remdesivir. It should, however, be noted that behind ICSRs reported in the described situation usually lies a deliberate clinical suspicion of an ADR by the treating physician. The DA used by us to analyse the ICSR reporting patterns is for the most part a method suitable to aid in the detection of new ADR hypotheses when qualitative methods are unfeasible, although in this case the results were promising in terms of accordance with the respective labelling. In conclusion, ICSR data and DA should primarily be used for hypotheses generation rather than to draw firm conclusions on causality or to deduct frequencies or incidences. Along the same lines, the described medicine use patterns may not be generalizable, although findings do appear to be in line with other sources of information $[34,35]$. One shortcoming was the lower completeness score of the remdesivir and tocilizumab ICSRs compared with all COVID-19-specific medicines. This was unfortunate but not surprising given that reports came mainly from the EU and the Americas, which do not typically include informative narratives (one factor of the completeness score) when shared into VigiBase. Further analysis of the narratives might shed further light on our findings. 


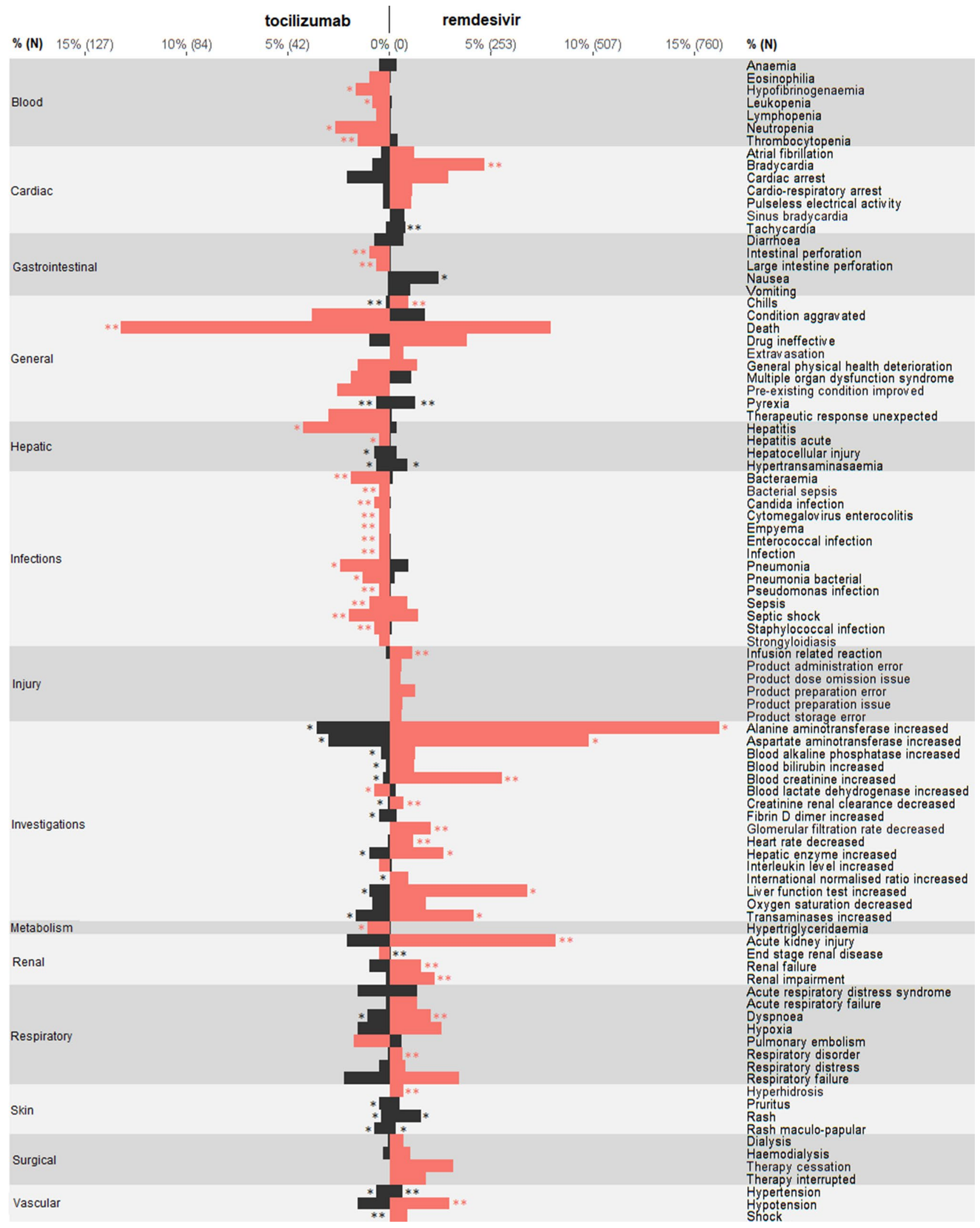


4Fig. 3 Plot showing the frequency of all preferred terms (PTs) for tocilizumab (left) and remdesivir (right) where the medicine is reported as suspected or interacting and where the PTs are reported in at least $0.5 \%$ of the Individual Case Safety Reports (ICSRs) for one of the medicines. The scales (top) are denoted in relative frequency per PT with frequency counts in parentheses. PTs are ordered alphabetically within their respective MedDRA System Organ Classes, abbreviated, far left. PTs disproportionately reported for the respective medicine in comparison with all other COVID-19 reporting (i.e. those with $\mathrm{IC}_{025 \text { COVID }}>0$ ) are marked in red. ICSRs with both remdesivir and tocilizumab reported as suspected or interacting are excluded. *The PT is included as an acknowledged adverse drug reaction (ADR) in the EU labelling for the related medicine. **The PT is included in relation to safety issues (e.g. warnings) in the EU labelling for the related medicine. A number of less meaningful PTs are omitted from the plot (see Supplementary Table 7 in the Electronic Supplementary Material)

\section{Conclusion}

In this study we have widened the use of data from global spontaneous reporting of ADRs. The underlying reasoning is that, although the first aim of including information in the ICSRs is to facilitate causality assessment, there is something to be gained from the mere descriptive overview of such information, especially in this particular historical moment. For instance, in the context of a lack of registry analyses and observational studies, a description of reported co-medications can give a first rough impression of clinical use of medicines during the first year of the COVID-19 pandemic.

Moreover, by using an indication-focused disproportionality analysis, together with the use of the comparator tocilizumab with a known safety profile, we were able to suggest severe liver dysfunction, severe kidney injury, medication errors and cardiac events as safety concerns for remdesivir. The observation that disproportionality analyses for remdesivir and a comparator are consistent with what is already known supports further investigation into the unexpected findings and those that appear to add to what is known. The causal hypotheses raised here should be further explored by the analysis of informative narratives and the motivated causality assessment by the reporter, when available.

Supplementary Information The online version contains supplementary material available at https://doi.org/10.1007/s40264-021-01091-x.

Acknowledgements The authors are indebted to the national centres that make up the WHO Programme for International Drug Monitoring and contribute ICSRs to VigiBase. However, the opinions and conclusions of this study are not necessarily those of the various centres nor of the WHO. The MedDRA ${ }^{\circledR}$ trademark is registered by IFPMA on behalf of ICH.

\section{Declarations}

Conflicts of interest Elena Rocca, Oskar Gauffin, Ruth Savage, Sara Hedfors Vidlin and Birgitta Grundmark declare no conflicts of interest.
Funding No external sources of funding were used to assist in the preparation of this study. Funding for the study being published with open access has been provided by the Uppsala Monitoring Centre.

Ethics approval Not applicable.

Consent to participate Not applicable.

Consent for publication Not applicable.

Availability of data and material The datasets generated and analysed during the current study are not publicly available due to agreements between contributors of data to the database used (VigiBase) and the custodian of this database. National centres (mainly national drug regulatory authorities) constituting the WHO Programme for International Drug Monitoring (PIDM) contribute data to VigiBase and the Uppsala Monitoring Centre is the custodian in its capacity as the WHO Collaborating Centre for International Drug Monitoring. Some subsets of the data may be available from the corresponding author on reasonable request.

Code availability The code used for the current study is not provided.

Author contributions BG, RS and OG conceived of the presented idea. All authors designed and developed the study together. OG and SV performed the algorithm development and the computations. All authors discussed results and contributed to the writing of the final manuscript. All authors read and approved the final version of the manuscript.

Open Access This article is licensed under a Creative Commons Attribution-NonCommercial 4.0 International License, which permits any non-commercial use, sharing, adaptation, distribution and reproduction in any medium or format, as long as you give appropriate credit to the original author(s) and the source, provide a link to the Creative Commons licence, and indicate if changes were made. The images or other third party material in this article are included in the article's Creative Commons licence, unless indicated otherwise in a credit line to the material. If material is not included in the article's Creative Commons licence and your intended use is not permitted by statutory regulation or exceeds the permitted use, you will need to obtain permission directly from the copyright holder. To view a copy of this licence, visit http://creativecommons.org/licenses/by-nc/4.0/.

\section{References}

1. World Health Organization. Descriptive analysis of COVID-19-related spontaneous reports from VigiBase: interim results. 2020. https://www.who.int/medicines/regulation/medicines-safety/ COVID19-PV-update4.pdf?ua=1. Accessed 1 June 2020.

2. Machhi J, Herskovitz J, Senan AM, Dutta D, Nath B, Oleynikov $\mathrm{MD}$, et al. The Natural history, pathobiology, and clinical manifestations of SARS-CoV-2 infections. J Neuroimmune Pharmacol. 2020;15(3):359-386.

3. Yang J, Zheng Y, Gou X, Pu K, Chen Z, Guo Q, et al. Prevalence of comorbidities and its effects in patients infected with SARSCoV-2: a systematic review and meta-analysis. Int J Infect Dis IJID Off Publ Int Soc Infect Dis. 2020;94:91-5.

4. Jin J-M, Bai P, He W, Wu F, Liu X-F, Han D-M, et al. Gender differences in patients with COVID-19: focus on severity and mortality. Front Public Health [Internet]. 2020;8:152.

5. Osuchowski MF, Aletti F, Cavaillon J-M, Flohé SB, GiamarellosBourboulis EJ, Huber-Lang M, et al. SARS-CoV-2/COVID-19: 
Evolving reality, global response, knowledge gaps, and opportunities. Shock. 2020;54(4):416-37.

6. European Medicines Agency. Summary of product characteristics for Veklury. 2020. https://www.ema.europa.eu/en/documents/ other/veklury-product-information-approved-chmp-25-june-2020pending-endorsement-european-commission_en.pdf. Accessed 21 Sept 2020.

7. Jorgensen SCJ, Kebriaei R, Dresser LD. Remdesivir: review of pharmacology, pre-clinical data, and emerging clinical experience for COVID-19. Pharmacother J Hum Pharmacol Drug Ther. 2020;40:659-71.

8. Elsawah HK, Elsokary MA, Abdallah MS, ElShafie AH. Efficacy and safety of remdesivir in hospitalized Covid-19 patients: Systematic review and meta-analysis including network metaanalysis. Rev Med Virol. 2021;31(4):e2187.

9. Consortium WST. Repurposed antiviral drugs for Covid19 - interim WHO solidarity trial results. N Engl J Med. 2021;384:497-511. https://doi.org/10.1056/NEJMoa2023184.

10. Beigel JH, Tomashek KM, Dodd LE, Mehta AK, Zingman BS, Kalil AC, et al. Remdesivir for the treatment of Covid-19-final report. N Engl J Med. 2021;384(9):795-807. https://doi.org/10. 1056/NEJMoa2031994.

11. Kaka AS, MacDonald R, Greer N, Vela K, Duan-Porter W, Obley A, et al. Major update: remdesivir for adults with COVID-19: a living systematic review and meta-analysis for the American College of Physicians Practice Points. Ann Intern Med. 2021;174(5):663-72. https://doi.org/10.7326/M20-8148.

12. Saint-Raymond A, Sato J, Kishioka Y, Teixeira T, Hasslboeck C, Kweder SL. Remdesivir emergency approvals: a comparison of the U.S., Japanese, and EU systems. Expert Rev Clin Pharmacol. 2020;13:1095-101.

13. Staff R. FACTBOX-Countries where remdesivir is approved or supported for treating COVID-19. Reuters. $2020 \mathrm{Jul}$ 10. https:// www.reuters.com/article/healthcoronavirus-gilead-remdesiviridUSL4N2EA2LZ. Accessed 20 Jan 2021.

14. Eastman RT, Roth JS, Brimacombe KR, Simeonov A, Shen M, Patnaik S, et al. Remdesivir: a review of its discovery and development leading to emergency use authorization for treatment of COVID-19. ACS Cent Sci. 2020;6:672-83.

15. Gérard AO, Laurain A, Fresse A, Parassol N, Muzzone M, Rocher F, et al. Remdesivir and acute renal failure: a potential safety signal from disproportionality analysis of the who safety database. Clin Pharmacol Ther. 2021;109:1021-4.

16. Touafchia A, Bagheri H, Carrié D, Durrieu G, Sommet A, Chouchana $\mathrm{L}$, et al. Serious bradycardia and remdesivir for coronavirus 2019 (COVID-19): a new safety concerns. Clin Microbiol Infect Off Publ Eur Soc Clin Microbiol Infect Dis. 2021;27(5):791. e5-791.e8. https://doi.org/10.1016/j.cmi.2021.02.013.

17. Chouchana L, Preta L-H, Tisseyre M, Terrier B, Treluyer J-M, Montastruc F. Kidney disorders as serious adverse drug reactions of remdesivir in coronavirus disease 2019: a retrospective casenoncase study. Kidney Int. 2021;99:1235-6.

18. Charan J, Kaur RJ, Bhardwaj P, Haque M, Sharma P, Misra S, et al. Rapid review of suspected adverse drug events due to remdesivir in the WHO database; findings and implications. Expert Rev Clin Pharmacol. 2021;14:95-103.

19. Lindquist M. VigiBase, the WHO Global ICSR database system: basic facts. Ther Innov Regul Sci. 2008;42:409-19. https://doi. org/10.1177/009286150804200501.
20. Behera SK, Das S, Xavier AS, Velupula S, Sandhiya S. Comparison of different methods for causality assessment of adverse drug reactions. Int J Clin Pharm. 2018;40:903-10.

21. Zekarias A, Watson S, Vidlin SH, Grundmark B. Sex differences in reported adverse drug reactions to COVID-19 drugs in a global database of individual case safety reports. Drug Saf. 2020;43(12):1309-14.

22. Oldenburg CE, Doan T. Azithromycin for severe COVID-19. The Lancet Elsevier. 2020;396:936-7.

23. Bergvall T, Norén GN, Lindquist M. vigiGrade: a tool to identify well-documented individual case reports and highlight systematic data quality issues. Drug Saf. 2014;37:65-77.

24. NIH. Clinical Spectrum of SARS-CoV-2 Infection. COVID-19 Treat. Guidel. 2021. https://www.covid19treatmentguidelines.nih. gov/overview/clinical-spectrum/. Accessed 24 Feb 2021.

25. Norén GN, Hopstadius J, Bate A. Shrinkage observed-to-expected ratios for robust and transparent large-scale pattern discovery. Stat Methods Med Res. 2013;22:57-69.

26. Grundmark B, Holmberg L, Garmo H, Zethelius B. Reducing the noise in signal detection of adverse drug reactions by standardizing the background: a pilot study on analyses of proportional reporting ratios-by-therapeutic area. Eur J Clin Pharmacol. 2014;70:627-35. https://doi.org/10.1007/s00228-014-1658-1.

27. European Medicines Agency. Summary of product characteristics for Roactemra 2021. https://www.ema.europa.eu/en/documents/ product-information/roactemra-epar-product-information_en.pdf Accessed23 Feb 2021.

28. R Core Team. R: a language and environment for statistical computing. R Foundation for Statistical Computing, Vienna, Austria. R Proj. Stat. Comput. 2017. https://www.r-project.org/. Accessed 20 May 2021.

29. Council for International Organizations of Medical Sciences, editor. Reporting adverse drug reactions: definitions of terms and criteria for their use. Repr. Geneva: CIOMS; 2000.

30. McMahon DE, Peters GA, Ivers LC, Freeman EE. Global resource shortages during COVID-19: Bad news for low-income countries. PLoS Negl Trop Dis. 2020;14:e0008412.

31. COVID-19 Hospitalizations. Centers For Disease Control and Prevention. 2021. https://gis.cdc.gov/grasp/covidnet/COVID19_5. html. Accessed 23 Feb 2021.

32. Carothers C, Birrer K, Vo M. Acetylcysteine for the treatment of suspected remdesivir-associated acute liver failure in COVID19: a case series. Pharmacother J Hum Pharmacol Drug Ther. 2020;40:1166-71.

33. Centers for Disease Control and Prevention. Interim Clinical Guidance for Management of Patients with Confirmed Coronavirus Disease (COVID-19). Cent. Dis. Control Prev. 2020. https:// www.cdc.gov/coronavirus/2019-ncov/hcp/clinical-guidancemanagement-patients.html Accessed 1 Mar 2021.

34. Horby P, Lim WS, Emberson JR, Mafham M, Bell JL, RECOVERY Collaborative Group, et al. Dexamethasone in hospitalized patients with Covid-19. N Engl J Med. 2021;384:693-704.

35. Paolisso P, Bergamaschi L, D'Angelo EC, Donati F, Giannella $\mathrm{M}$, Tedeschi $\mathrm{S}$, et al. Preliminary experience with low molecular weight heparin strategy in COVID-19 patients. Front Pharmacol. 2020;6(11):1124. https://doi.org/10.3389/fphar.2020.01124.

36. Bilotta F, Guerra C, Badenes R, Lolli S, Rosa G. Short acting insulin analogues in intensive care unit patients. World J Diabetes. $2014: 5: 230-4$. 